\title{
Epigenetic Regulation of miR-129-2 Leads to Overexpression of PDGFRa and FoxP1 in Glioma Cells
}

\author{
Xiang-Yang Tian ${ }^{1 *}$, Ling Zhang ${ }^{2}$, Lai-Guang Sun ${ }^{1}$, Ming $\mathrm{Li}^{3 *}$
}

\begin{abstract}
miR-129-2 is frequently downregulated in multiple cancers. However, how it is silenced in cancers remains unclear. Here we investigated the expression profile and potential biological function of miR-129-2 in glioblastoma (GBM), the most common and lethal form of brain tumors in adults. We showed that miR-129-2 is lost in GBM patient specimens and cultured cell lines. miR-129-2 expression could be restored upon treatment with a histone deadetylase inhibitor (trichostatin A) but not a DNA methylation inhibitor (5-Aza-2'-deoxycytidine), and more profound effect was observed with the treatment of these two drugs in combination. Furthermore, forced expression of miR-129-2 repressed the expression of major oncogenic genes such as PDGFRa and Foxp1 in GBMs. Consistently, expression of miR-129-2 significantly inhibits GBM cell proliferation in vitro. These results reveal that miR-129-2 is epigenetically regulated and functions as a tumor suppressor gene in GBMs, suggesting it may serve as a potential therapeutic target for GBM treatment.
\end{abstract}

Keywords: Glioblastoma - microRNA-129 - epigenetic regulation

Asian Pac J Cancer Prev, 16 (14), 6129-6133

\section{Introduction}

MicroRNAs are small non-coding RNAs which are present in plants, animals and some viruses. The primary microRNAs are transcribed by RNA polymerase II and recognized and cleaved by the DGCR8 (DiGeorge syndrome critical region 8) protein complex to produce the 70-nt pre-microRNAs, which are then released from the nucleus into the cytoplasm, where the Dicer complex cleaves the loop region on the pre-microRNA hairpin to create the 18-22 bp double-stranded mature microRNAs (Gregory et al., 2004). Mature microRNAs are incorporated into a RNA-induced silencing complex (RISC) which interacts with mRNAs, leading to mRNA degradation or translation inhibition (Floyd et al., 2014).

MicroRNAs are highly conserved across species and involved in many biologic processes. In cancers, microRNA expression is frequently deregulated through multiple mechanisms such as genomic alterations DNA methylation, aberrant transcription, and defective microRNA processing (Calin et al, 2006; Ma et al., 2010). By regulating target genes expression, microRNAs play an important role in cancer initiation, maintenance, and progression (Calin et al., 2006).

micoRNA-129-2 (miR-129-2) has been reported to be frequently deregulated in many cancers, which include glioblastoma (GBM), the most common and deadly primary brain tumor (Furnari et al., 2015). miR-
129-2 could act as an oncogene or tumor suppressor gene depending on the cancer origin (Lu et al., 2013; Li et al., 2013). Therefore, the function of miR-129-2 in cancers remains controversial.

In this report, we sought to explore the expression profile of miR-129-2 and its potential biological function in GBM. Our data showed that the expression of miR-1292 is significantly downregulated in GBM specimens and cell lines. The downregulation of miR-129-2 is mainly through chromatin remodeling and DNA methylation. Functionally, restoration of miR-129-2 expression inhibits glioma cell proliferation partially through repressing of some major oncogenic genes, such as PDGFRa and Foxp1.

\section{Materials and Methods}

\section{Tissues and cells}

All glioma cells including U87, U178, U373, LN308, LN215, U251, A1207 and SNB19 were obtained from ATCC and cultured in Dulbecco's Modified Eagle Medium (DMEM) containing 10\% FBS. GBM tissues and neurospheres were isolated from fresh GBM patient samples with an agreement in our hospital.

\section{Quantitative real-time PCR}

The cells were treated with trichostatin A(TSA, histone deadetylase inhibitor, Sigma) or 5-Aza-2'-deoxycytidine (5-Aza, the DNA methylation inhibitor, Sigma) for 24

${ }^{1}$ Department of Neurosurgery, ${ }^{2}$ Medical Record Office, Xinxiang Central Hospital, Xinxiang, Henan Province, ${ }^{3}$ Experimental Center, the Second Affiliated Hospital of Soochow University, Suzhou, Jiangsu Province, China *For correspondence: m2liva@hotmail. com,13837314850@126.com 
Xiang-Yang Tian et al

hr prior to RNA isolation. cDNA was synthesized from $1 \mu \mathrm{g}$ of DNase I-treated RNA using the SuperScript III First-Strand Synthesis SuperMix for qPCR Kit (Life Technologies). The primersequences used to amplify hsapri-miR-129-2 were as described previously (Huang et al., 2009). Mature miRNA expression was determined using small RNA TaqMan assays according to manufacturer's instructions (Life Technologies). All reactions were done in triplicate. The expression of miR-129-2 was normalized using RNU48 (Applied Biosystems, Part No 4373383). The expression relative to RNU48 was determined using the $2^{-\Delta \mathrm{Ct}}$ method.

\section{Antibodies and Western blotting}

Western blotting was performed as described previously (Li et al., 2011). Briefly, the cells was lysed in RIPA buffer [50mM Tris ( $\mathrm{pH} 7.4$ ), $150 \mathrm{mM} \mathrm{NaCl}, 1 \%$ NP-40, $0.5 \%$ sodium deoxycholate, $0.1 \%$ SDS, with the addition of Protease Inhibitor Cocktail (Roche)]. The proteins samples were subjected to sodium dodecyl sulfate polyacrylamide gel electrophoresis (SDS-PAGE, 10\% acrylamide resolving gels). The Antibodies used in this study were as follows: anti-GAPDH, anti-FoxP1 and antiPDGFRa were obtained from Cell Signaling Technology, and the anti-Mouse/-Rabbit Secondary Antibodies were purchased from Santa Cruz Biotechnology.

\section{Transduction of miR-129-2 using a lentiviral system}

The pHAGE-CMV-eGFP-miR-129 construct was a gift of Dr. Jining Lü and described previously (Wu et al., 2010). Packaging of all lentiviral vectors was performed by co-transfection of the $293 \mathrm{~T}$ cell line with $5 \mu \mathrm{g}$ plasmids encoding the lentiviral backbone as well as tat, rev, gag/pol and vsv-g viral genes. Viral particles in the resulting supernatants were collected and concentrated by ultracentrifugation. Titers used in all experiments ranged from $0.5-1.5 \times 10^{9} \mathrm{pfu} / \mathrm{ml}$.

\section{Cell viability assay}

The cell viability was tested using WST-1 (Roche) assay as describe previously ( $\mathrm{Li}$ et al., 2011). Briefly, the cells were plated at 1500 cells/well in 96-well plates and cultured for the indicated time, and $10 \mu \mathrm{L}$ of WST-1 solution was added to each well, and the plates were incubated at $37^{\circ} \mathrm{C}$ for $4 \mathrm{~h}$, followed by absorbance measurement at 450 and $900 \mathrm{~nm}$ by using a GENios Pro microplate reader (Tecan).

\section{Statistical analysis}

All results are expressed as the mean \pm SD. Statistical analyses were performed by Student's t test. $\mathrm{P}<0.05$ was considered statistically significant.

\section{Results}

\section{miR-129 is silenced in glioblastoma}

We fist did in silico analysis of the miR-129 expression profile in various tissues (http://www.microrna.org). As shown in Figure 1A, miR-129 is highly expressed in brain as compared to other tissues. This led us to examine its expression level in glioblastoma. Using the Cancer
Genome Atlas (TCGA) database, it was found that miR129 is significantly downregulated in all the four subtypes (proneural, neural, classical and mesenchymal) of GBMs compared to the normal brain tissues (Figure 1B). To validate this, we did RT-qPCR to analyze the expression level of miR-129 in a collection of 18 GBM specimens, 9 culture cell lines and 9 newly isolated neurosphere lines. We found that the expression of miR-129 is significantly lower in the GBM patient samples as well as the cultured cells compared to the non-tumor tissues (Figure 1C).

miR-129-2 is epigenetically regulated by chromatin remodeling and DNA methylation.

Next we examined how miR-129 is frequently lost in GBMs. Since it has been reported that the promoter of
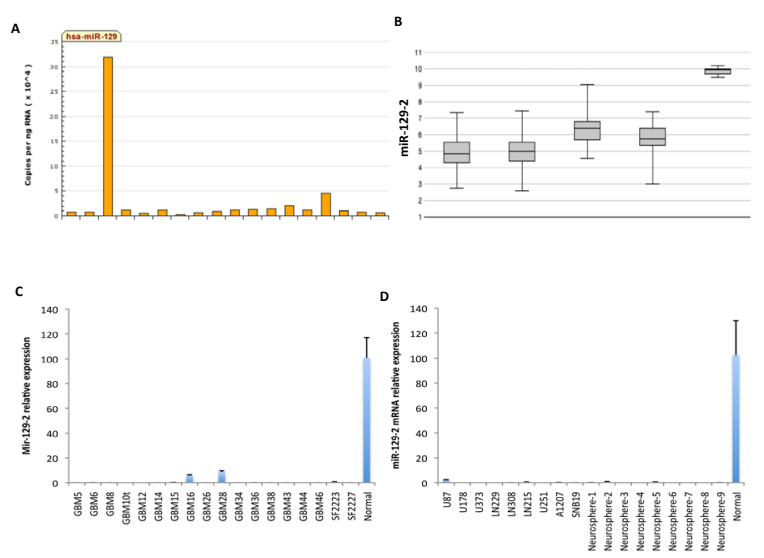

Figure 1. Brain Specific miR-129 is Silenced in GBMs. (A). In silico analysis of the mRNA expression profile of miR129 in various human tissues (http://www.microrna.org). (B). TCGA analysis of miR-129 mRNA level in the subtypes of GBMs. (C)qRT-PCR analysis of miR-129 mRNA expression in GBM specimens and normal brain tissues. (D) qRT-PCR analysis of miR-129 mRNA expression in GBM cell lines and neurospheres

A
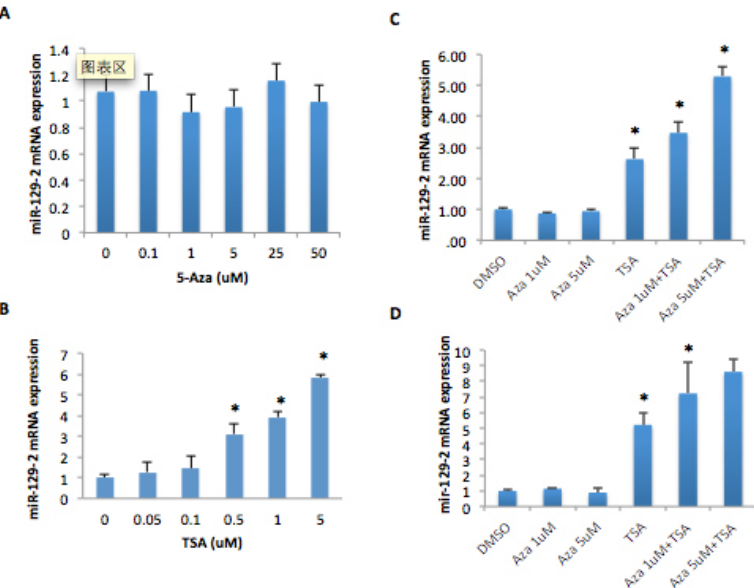

Figure 2. miR-129-2 is Epigenetically Regulated in GBM Cells. (A) Effect of 5-Aza on the miR-129-2 expression in T98G cells. The concentration of 5-Aza is indicated on the figure. (B) Effect of TSA on the miR-129-2 expression in T98G cells. The concentration of TSA is indicated on the figure. (C) Effect of TSA $(5 \mu \mathrm{M})$ and 5-Aza $(1$ and $5 \mu \mathrm{M})$ combination on the miR-219-2 expression in T98G cells. (D) Effect of TSA (5 $\mu \mathrm{M})$ and 5-Aza (1 and $5 \mu \mathrm{M})$ combination on the miR-129-2 expression in A1207 cells. * $p<0.05$ 
A

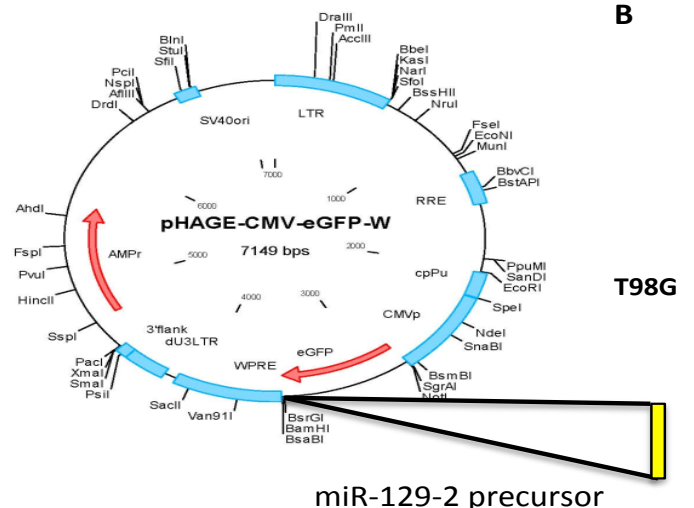

B

Vector control

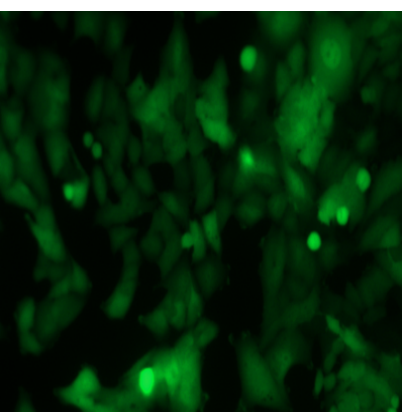

miR-129-2

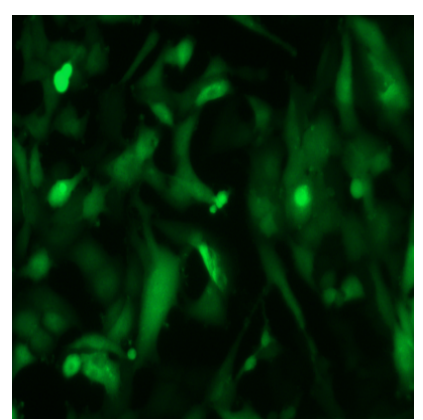

Figure 3. Lentiviral-mediated Transduction of miR-129-2 in GBM Cells. (A) Diagram of the lentiviral pHAGE-CMVeGFP-miR-129 construct. (B) Representative fluorescence image of the miR-129 overexpression in T98G cells

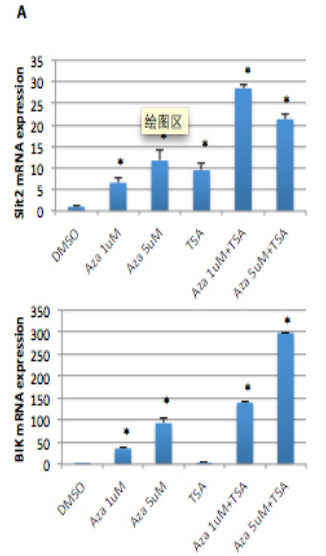

Supplemental Figure 1. Slit2 and BIK are Epigenetically Regulated in GBM Cells. (A) Effect of 5-Aza and TSA on the mRNA expression of Slit2 and BIK in T98G cells. (B) Effect of 5-Aza and TSA on the mRNA expression of Slit2 and BIK in A1207 cells $* p<0.05$

miR-129 is frequently methylated in other cancers (Huang et al. 2009), we determined whether this downregulation is mediated by epigenetic mechanisms. U87 cells were treated with a demethylating agent, 5-Aza prior to RTqPCR analysis. As shown in Figure 2A, there is no obvious change of the miR-129-2 expression with 5-Aza treatment at different concentration. To validate the quality of the chemical, we observed that 5-Aza treatment significantly upregulated the mRNA level of SLIT2 and BIK, two wellknown methylation-regulated gene (supplemental Figure 1). We then examined the effect of a histone deacetylase (HDAC) inhibitor, TSA, on the mRNA expression of miR129. It was found that miR-129-2 is dramatically induced by TSA in a dose dependent manner (Figure 2B). When these cells were treated with 5-Aza (1 and 5 $\mu \mathrm{M})$, TSA $(5 \mu \mathrm{M})$, or their combination, reactivation of miR-129-2 was observed in two independent cell lines (U87 and T98G) analyzed that were treated with TSA. More profound effects of this reexpression were found in these cell lines treated with the combination of TSA and 5-Aza (Figure 2C and 2D). These results suggest HDAC is involved in the regulation of miR-129-2 expression.

Restoration of miR-129-2 inhibits GBM cell proliferation To investigate the biological function of miR-129-2 in GBM, we elevated the expression level of miR-129-2 in T98G cells by lentiviral transduction of pHAGECMV-eGFP-miR-129-2 (Figure 3A). The expression of miR-129-2 in the cells was confirmed by GFP expression (Figure 3B). We then analyzed the effect of miR-1292 expression on the cell viability. Overexpression of miR-129-2 significantly suppressed the growth of T98G cells (Figure 4A). Similar data was obtained in another independent cell line A1207 (Figure 4B).

\section{miR-129-2 regulates PDGFRA and FoxPl expression in} GBM cells

Finally we explored the potential mechanism by which miR-129-2 inhibits GBM cell proliferation. We first predicted the potential target genes of miR-129-2 using MicroRNA targets prediction tools (http://www. microrna.org). Interestingly two major oncogenic genes, PDGFRA (platelet-derived growth factor receptor alpha) and FoxP1 (Forkhead Box P1), were found to be potential targets of miR-129-2 (Figure 5A). To confirm this, we differentially overexpressed miR-129-2 in T98G and A1207 cells. We observed significant repression of both PDGFRa and FoxP1 protein levels in these two different cell lines when differential dose of miR-129-2 was taken into account (Figure 5B).

\section{Discussion}

In this study we demonstrated that expression of miR-129-2 is lost in GBMs mainly through histone deacetylation, and DNA methylation may be also involved in this epigenetic regulation. Functionally, restoration of miR-129-2 expression significantly reduces GBM cell proliferation. Our data also suggested that PDGFRA and FOXP1 may be novel target genes of miR-129-2 in GBMs and be involved in miR-129-2-mediated GBM cell proliferation.

It has been reported that miR-129 is silenced and functions as a tumor suppressor gene in multiple cancers, including gastric cancer, lung cancer, neuroendocrine tumor, endometrial cancer, hepatocellular carcinoma, colorectal cancer, cervical cancer, medullary thyroid carcinoma, and renal cell carcinoma (Huang et al., 2009; Chen et al., 2013; Karaayvaz et al., 2013; Lu et al., 2013; 


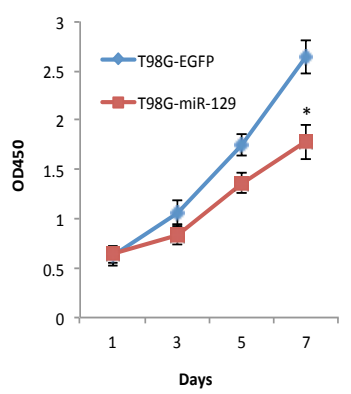

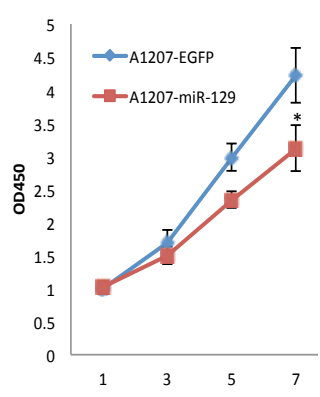

Figure 4. Restoration of miR-129-2 Expression Inhibits GBM Cell Proliferation. Effect of miR-129 on T98G (A) and A1207 cells (B) was analyzed using WST-1 assay. $p<0.05$

A

hsa-miR-129-5p/FOXP1 Alignment:

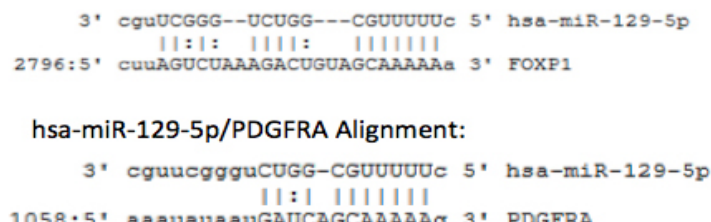

B

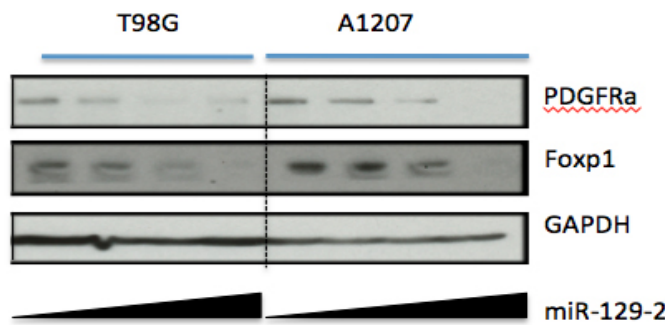

Figure 5. miR-129-2 Expression Reduces PDGFRa and FoxP1 Expression in GBM Cells. (A) PDGFRa and FoxP1 are potential targets of miR-129-2 ((http://www.microrna.org). (B) Effect of miR-129-2 restoration on the PDGFRa and FoxP1 protein expression in T98G and A1207 cells. Western blot was performed. GAPDH is shown as loading control. The titer of the miR-129 lentivirus used is $(0.25,0.5,1.0,1.5) \times 10^{9} \mathrm{Pfu} / \mathrm{ml}$

Zhang et al., 2013; Dossing et al., 2014; Fesler et al., 2014; Liu et al., 2014). In contrast, some other report showed that miR-129 is upregulated in laryngeal squamous cell carcinoma and functions as an oncogene in LSCC (Li et al., 2013). We presented evidence that histone deacetylation has a larger role in the inhibition of miR-129 expression in the human GBM cells than does DNA methylation. This is demonstrated by the greater effect by the HDAC inhibitor TSA on gene expression than of the DNMT inhibitor 5-Aza. It is also demonstrated that the synergy between 5-Aza and TSA on miR-129-2 expression in these cells by performing quantitative real-time RT-PCR. These data suggested that the interaction or cooperation of chromatin modification and DNA methylation may be involved in miR-129 expression.

Since miR-129 is silenced in GBMs, gain-of-function study has been performed to examine its biological function in vitro. Lentivirus restoration of miR-129-2 expression significantly reduces GBM cell growth, suggesting that miR-129-2 has anti-tumor function.
Consistent with this, our bioinformatics analysis indicated that two major oncogenes, PDGFRa and FoxP1, are predicted target genes of miR-129-2. Our data showed that forced expression of miR-129-2 is able to reduce the expression of both PDGFRa and FoxP1 in GBM cell lines, which may partially explain how miR-129 functions as a tumor suppressor in this particular cancer. PDGFRa was the third of the top 11 amplified or overexpressed genes in clinical GBM specimens and plays a key role in proneural subtype of GBM and secondary GBMs (Liu et al., 2011). A recent report demonstrated that FoxP1 was sufficient to increase glioma growth and that high FOXP1 expression predicts poor survival in patients with GBM (Gomez et al., 2014).Our results suggested that silencing of miR-129 may contribute to the elevated level of these two genes. Further work will be required to show these two genes are direct targets of miR-129-2 in GBM.

In conclusion, our findings support an idea that epigenetically mediated silencing of miR-129-2 contributes to oncogene activation in cancer cells. Therefore, the combined epigenetic and miRNA-based therapies might be an option for future treatments in cancer patients.

\section{Acknowledgements}

This work was supported by National Natural Sciences Foundation of China (81172401).

\section{References}

Calin GA, Croce CM (2006). MicroRNA signatures in human cancers. Nat Rev Cancer, 6, 857-66.

Chen X, Zhang L, Zhang T, et al (2006). Methylation-mediated repression of microRNA 129-2 enhances oncogenic SOX4 expression in HCC. Liver Int, 3, 476-86.

Dossing KB, BinderuP T, Kaczkowski B, et al (2014). Down-Regulation of miR-129-5p and the let-7 Family in Neuroendocrine Tumors and Metastases Leads to UpRegulation of Their Targets Egr1, G3bp1, Hmga2 and Bach1. Genes (Basel), 6, 1-21

Fesler A, Zhai H, Ju J (2014). miR-129 as a novel therapeutic target and biomarker in gastrointestinal cancer. Onco Targets Ther, 7, 1481-5.

Floyd D, Purow B (2014). Micro-masters of glioblastoma biology and therapy: increasingly recognized roles for microRNAs. Neuro Oncol, 16, 622-7.

Furnari FB, Cloughesy TF, Cavenee WK, et al (2015). Heterogeneity of epidermal growth factor receptor signalling networks in glioblastoma. Nat Rev Cancer, 15, 302-10.

Gregory RI, Yan KP,Amuthan G, et al (2014). The Microprocessor complex mediates the genesis of microRNAs. Nature, $\mathbf{4 3 2}$, 235-40.

Gomez GG, Volinia S, Croce CM, et al (2014). Suppression of microRNA- 9 by mutant EGFR signaling upregulates FOXP1 to enhance glioblastoma tumorigenicity. Cancer Res, 74, 1429-39.

Huang YW, Liu JC, Deatherage DE, et al (2009). Epigenetic repression of microRNA-129-2 leads to overexpression of SOX4 oncogene in endometrial cancer. Cancer Res, 69, 9038-46.

Karaayvaz M, Zhai H, Ju J. (2013). miR-129 promotes apoptosis and enhances chemosensitivity to 5-fluorouracil in colorectal cancer. Cell Death Dis, 4, 659.

Li M, Mukasa A, Inda MM, et al (2011). Guanylate binding 

protein 1 is a novel effector of EGFR-driven invasion in glioblastoma. J Exp Med, 208, 2657-73.

Li M, Tian L, Wang L, et al (2013). Down-regulation of miR$129-5 p$ inhibits growth and induces apoptosis in laryngeal squamous cell carcinoma by targeting APC. PLoS One, $\mathbf{8}$, 77829.

Liu KW, Hu B, Cheng SY (2011). Platelet-derived growth factor receptor alpha in glioma: a bad seed. Chin J Cancer, 30, 590-602.

Liu MX, Zhou KC, Cao Y. MCRS1 overexpression, which is specifically inhibited by miR-129*, promotes the epithelialmesenchymal transition and metastasis in non-small cell lung cancer. Mol Cancer, 13, 245.

Lu CY, Lin KY, Tien MT, et al (2013). Frequent DNA methylation of MiR-129-2 and its potential clinical implication in hepatocellular carcinoma. Genes Chromosomes Cancer, 52, 636-43.

Ma L, Young J, Prabhala H, Pan E, et al (2010). miR-9, a MYC/ MYCN-activated microRNA, regulates E-cadherin and cancer metastasis. Nat Cell Biol, 12, 247-56.

Wu J, Qian J, Li C, et al (2010). miR-129 regulates cell proliferation by downregulating Cdk6 expression. Cell Cycle, 9, 1809-18.

Zhang J, Li S, Yan Q, et al (2013). Interferon- $\beta$ induced microRNA-129-5p down-regulates HPV-18 E6 and E7 viral gene expression by targeting SP1 in cervical cancer cells. PLoS One, 8, 81366. 